REFERENCES

1. Hoffman PC, Dixon RE. Control of influenza in the hospital. Ann Intern Med 1977;87:725-728.

2. Hall CB. Nosocomial viral respiratory infections: perennial weeds on pediatric wards. Am J Med 1981;70:670-676.

3. Weingarten S, Friedlander M, Rascon D, Ault M, Morgan M, Meyer RD. Influenza surveillance in an acute-care hospital. Arch Intern Med 1988;148:113-116.

4. Van Voris LP, Belshe RB, Shaffer JL. Nosocomial influenza B virus infection in the elderly. Ann Intern Med 1982;96:153-158.

5. Yassi A, McGill M, Holton D, Nicolle L. Morbidity, cost and role of health care worker transmission in an influenza outbreak in a tertiary care hospital. Can J Infect Dis 1993;4:52-56.

6. Hammond GW, Cheang M. Absenteeism among hospital staff during an influenza epidemic: implications for immunoprophylaxis. Can Med Assoc I 1984;131:449-452

7. Yassi A, Kettner J, Hammond G, Cheang M, McGill M. Effectiveness and cost-benefit of an influenza vaccination program for health care workers. Can J Infect Dis 1991;2:101-108.

8. Elder AG, O'Donnell B, McCruden EAB, Symington IS, Carman WF. Incidence and recall of influenza in a cohort of Glasgow healthcare workers during the 1993-4 epidemic: results of serum testing and questionnaire. $B M J$ 1996;313:1241-1242.

9. Fedson DS, Hannoun C, Leese J, Sprenger MJ, Hampson AW, BroJorgensen $\mathrm{K}$, et al. Influenza vaccination in 18 developed countries, 1980 1992. Vaccine 1995;13:623-627.

10. Fleming DM, Zambon M, Bartelds AIM, de Jong JC. The duration and magnitude of influenza epidemics. Eur J Epidemiol 1999;15:467-473.

\title{
Efficacy of Intranasal Influenza Vaccine
}

\section{Gina Pugliese, RN, MS Martin S. Favero, PhD}

Influenza virus is a major cause of illness, disruption to daily life, and increased use of health care in all age groups. Nichols and colleagues recently conducted a study to assess the safety and effectiveness of intranasally administered trivalent, live, attenuated influenza virus (LAIV) vaccine for reducing illness, absenteeism, and healthcare use among healthy, working adults.

A total of 4,561 healthy, working adults aged 18 to 64 participated in a randomized, double-blind, placebocontrolled trial conducted from September 1997 through March 1998 in 13 centers across the United States. Participants were recruited through health insurance plans, at work sites, and from the general population and were randomized 2:1 to receive intranasally administered trivalent LAIV vaccine $(n=3,041)$ or placebo $(n=1,520)$ in the fall of 1997.

Recipients of LAIV vaccine were as likely to experience one or more febrile illnesses as placebo recipients during peak outbreak periods $(13.2 \%$ for vaccine vs $14.6 \%$ for placebo; $P=.19$ ). However, vaccination significantly reduced the numbers of severe febrile illnesses (18.8\% reduction) and febrile upper respiratory tract illnesses (23.6\% reduction). Vaccination also led to fewer days of illness across all illness syndromes $(22.9 \%$ reduction for febrile illnesses; $27.3 \%$ reduction for severe febrile illnesses), fewer days of work lost $(17.9 \%$ reduction for severe febrile illnesses; $28.4 \%$ reduction for febrile upper respiratory tract illnesses), and fewer days with healthcare provider visits $(24.8 \%$ reduction for severe febrile illnesses; $40.9 \%$ reduction for febrile upper respiratory tract illnesses). Use of prescription antibiotics and over-the-counter medications was also reduced across all illness syndromes. Vaccine recipients were more likely to experience runny nose or sore throat during the first 7 days after vaccination, but serious adverse events between the groups were not significantly different. The match between the type $\mathrm{A}(\mathrm{H} 3 \mathrm{~N} 2)$ vaccine strain and the predominant circulating virus strain (A/Sydney/05/97[H3N2]) for the 1997-98 season was poor, suggesting that LAIV provided substantial cross-protection against this variant influenza $A$ virus strain.

The researchers concluded that intranasal trivalent LAIV vaccine was safe and effective in healthy, working adults in a year in which a drifted influenza A virus predominated.
In an accompanying editorial by Poland and Couch, it is noted that $70 \%$ of study participants self-administered the vaccine. This suggests a potential improvement in the ability to prevent influenza transmission by the widespread means of painlessly self-administering a vaccine whenever it is convenient. A further advantage would be the ability to obtain the vaccine over the counter, eliminating the cost and inconvenience barriers currently associated with mass influenza immunization programs. Additionally, these factors would have tremendous benefits for the development of a vaccine-based program to prevent pandemic influen$z a$, assuming such a vaccine could be manufactured and distributed in sufficient quantity and in a timely manner. Such a vaccine also would be appealing for preventing epidemic influenza transmission by mass immunization among the most efficient transmitters of disease, school-aged children.

FROM: Nichol KL, Mendelman PM, Mallon KP, Jackson LA, Gorse GJ, et al. Effectiveness of live, attenuated intranasal influenza virus vaccine in healthy, working adults: a randomized controlled trial. JAMA 1999;282:137-144.

Poland GA, Couch R. Intranasal influenza vaccine: adding to the armamentarium for influenza control. JAMA 1999;282:182-184. 